Pacific Journal of Mathematics

GENERALIZED INVERSES IN REGULAR RINGS 


\title{
GENERALIZED INVERSES IN REGULAR RINGS
}

\author{
Thomas R. SAvage
}

\begin{abstract}
Motivated by I. Kaplansky's theorem on one-sided inverses in rings, we consider, for a given nonzero element $a$ in a regular ring, the number of solutions $x$ to (i) $a=a x a$, (ii) $a=a x a$ and $x=x a x$, and (iii) $a=a x a$ with $x$ invertible. Our main result: If a prime regular ring $R$ contains an element $a$ for which the number of solutions to (i), (ii), or (iii) is finite and greater than one, then $R$ is a matrix ring over a finite field. Complete descriptions are given of those regular rings for which the number of solutions to (i), (ii), or (iii) is always one and those for which the number is always finite.
\end{abstract}

1. Introduction. The theorem of Kaplansky mentioned above asserts that in any ring with identity an element with a one-sided inverse either is invertible or has infinitely many one-sided inverses. Elegant, elementary proofs of this theorem have been given by N. Jacobson [8] and by C. W. Bitzer [2]. This paper proceeds in the spirit of Bitzer's method of proof, which is a counting argument: if $a$ has a right inverse then $a x=1$ has either exactly one solution or infinitely many solutions. Analogously, we present conditions which guarantee that, for a nonzero element $a$ in a regular ring, the number of solutions to (i) $a=a x a$, (ii) $a=a x a, x=x a x$, or (iii) $a=a x a$ with $x$ invertible, is either exactly one or infinite. For example, if the ring has no integral torsion or if the ring is prime, regular, and infinite, then (i), (ii), or (iii) always has either exactly one solution or infinitely many solutions. (For (iii), we need to require that there is at least one solution.) Subsequently, we characterize those regular rings for which the number of solutions to (i), (ii), or (iii) is always finite. For example, if the number of solutions to (iii) is finite and larger than one for each nonzero $a$ in $R$, then $R$ is a division ring, a Boolean ring, a matrix ring over a finite field, or a direct sum of two rings of the latter two types.

Portions of this paper appear in the author's doctoral dissertation written at the Claremont Graduate School under the supervision of Professor Melvin Henriksen. The author extends his warm thanks to Professor Henriksen for his generous help and encouragement throughout the preparation of this paper.

2. Preliminaries. We begin with some terminology relating to regular rings. Throughout $R$ denotes an associative ring. An element $a$ in $R$ is (von Neumann) regular if $a=a x a$ has a solution 
in $R$ and any such solution $x$ is called a generalized inverse of $a$. Following G. Ehrlich in [4], the element a in $R$ is called unit regular if $R$ has an identity and $a$ has an invertible generalized inverse. A reflexive inverse of $a$ is a ring element $x$ such that $a=a x a$ and $x=x a x$. Every regular element possesses a reflexive inverse, for if $a=a x a$ then $y=x a x$ is a reflexive inverse of $a$. The ring $R$ is regular (resp. unit regular) if each of its elements is regular (resp. unit regular). Finally, $R$ is strongly regular (see Arens and Kaplansky [1]) if for each $a$ in $R$ there is an $x$ in $R$ such that $a=$ $a^{2} x$. Strong regularity always implies regularity and, in the presence of an identity element, strong regularity implies unit regularity which implies regularity. See [13], [1], [11], [4], [7] for greater detail.

3. Main results. The first results of this section characterize those ring elements which possess a unique generalized inverse, reflexive inverse, or invertible generalized inverse. Some of these results are certainly known and are included here for easy reference.

Lemma 3.1. For a nonzero regular element a of a ring $R$, the following statements are equivalent:

(i) a has a unique generalized inverse.

(ii) a is neither a right nor a left divisor of zero.

(iii) $R$ has an identity and $a$ is a unit.

Proof. (i) implies (ii). If $x$ is the unique generalized inverse of $a$ and if $a z=0$ or $z a=0$, then $a(x+z) a=a$. By uniqueness, $x=x+z$, whence $z=0$.

(ii) implies (iii). Suppose $a$ is neither a right nor a left divisor of zero. Choose an $x$ with $a=a x a$. For any $y$ in $R$ we have $a(y-x a y)=0=(y-y a x) a$ and therefore, $x a y=y=y a x$. Thus $x a$ is a left identity and $a x$ is a right identity for $R$. Hence, $e=$ $a x=x a$ is the identity for $R$ and $a$ is clearly a unit.

(iii) implies (i). If $R$ has the identity 1 and $a$ is a unit, then $a=a x a$ implies $a x=1=x a$, so $x=a^{-1}$.

Proposition 3.2. [12, Exercise 26, p. 10] A nonzero ring $R$ is a division ring if and only if each nonzero element of $R$ has a unique generalized inverse.

Proof. This is evident from Lemma 3.1.

LEMMA 3.3. If a is a regular element of the ring $R$, then the following statements are equivalent: 
(i) a has a unique reflexive inverse.

(ii) There is an $x$ in $R$ such that $a=a x a$ and both ax and xa are central idempotents.

(iii) If $a=a y a$, then $a y=y a$.

(iv) If $a=a y a=a z a$, then $a y=a z=z a=y a$.

Proof. (i) implies (ii). Let $x$ be the unique element of $R$ for which $a=a x a$ and $x=x a x$. For any $y$ in $R$ the elements $x+y-$ $x a y$ and $x+y-y a x$ are generalized inverses of $a$ and hence,

$$
\begin{aligned}
x & =(x+y-x a y) a(x+y-x a y)=x+y a x-x a y a x \\
& =(x+y-y a x) a(x+y-y a x)=x+x a y-x a y a x .
\end{aligned}
$$

Therefore, $y a x=x a y$ for every $y$ in $R$. Letting $y$ be $a x$ and $x a$ successively, we obtain $a x=x a^{2} x=x a$. So (ii) holds.

(ii) implies (iii). Choose an $x$ with $a=a x a$ and both $a x$ and $x a$ in the center. Then

$$
a x=a(x a) x=(a x)(x a)=(x a)(a x)=x(a x) a=x a .
$$

Hence, if $a=a y a$, then

$$
a y=(a x)(a y)=(a y a) x=a x=x a=x(a y a)=(y a)(x a)=y a .
$$

(iii) implies (iv). If $a=a y a=a z a$, then by (iii),

$a y=y a=y(a z a)=(y a)(z a)=(a y)(a z)=(a y a) z=a z=z a$.

(iv) implies (i). If $y$ and $z$ are reflexive inverses of $a$,

then $y=y a y=y a z=z a z=z$.

It is known that a ring $R$ is strongly regular if and only if $R$ is regular and every idempotent of $R$ is central, see [1]. Therefore, the next result is an immediate consequence of Lemma 3.3.

Proposition 3.4. A ring $R$ is strongly regular if and only if each element of $R$ has a unique reflexive inverse.

To obtain the corresponding results for unit regularity, we employ the following characterization of unit regularity which is due to Kaplansky and which appears in Henriksen [7, Prop. 8]: If $R$ is regular with identity and $a$ is in $R$, then $a$ is unit regular if and only if whenever $a R+b R=R$ there is $a t$ in $R$ such that $a+$ $b t$ is a unit. By applying this result to the opposite ring of $R$, it is clear that a being unit regular is equivalent to: $R a+R b=R$ implies there is an $s$ such that $a+s b$ is a unit.

Lemma 3.5. Suppose $R$ is a regular ring with identity and a 
is a unit regular element of $R$. If $x$ is any reflexive inverse of a, then there are invertible generalized inverses $p$ and $q$ of $a$ such that $x=p a q$.

Proof. Suppose $a=a x a$ and $x=x a x$. Then $a R+(1-a x) R=$ $R$, so the result quoted above guarantees $a t$ in $R$ such that $a+$ $(1-a x) t=q^{-1}$ is a unit. Left multiplication by $a x$ and right multiplication by $q$ in this equation yield $a q=a x$. A similar argument using $R a+R(1-x a)=R$ shows there is a unit $p$ in $R$ such that $p a=x a$. Clearly, $p$ and $q$ are generalized inverses of $a$ and $x=x a x=x a q=p a q$.

LEMMA 3.6. Suppose $R$ is a regular ring with identity and a in $R$ has a unique invertible generalized inverse $u$. Then a has a unique reflexive inverse and $2 a u=2$. In particular, if $2^{-1} \in R$ then $a$ is a unit.

Proof. If $u$ is the unique invertible generalized inverse of $a$, then Lemma 3.5 implies that $u a u$ is the unique reflexive inverse of a. By Lemma 3.3, we have $a u=u a$ is in the center of $R$. It follows easily that $1-a u+u a u$ and $a u-1+u a u$ are units with inverses $1-a u+a$ and $a u-1+a$, respectively. Since each of these units is a generalized inverse of $a$, we deduce that $1-a u+$ $u a u=a u-1+u a u$. Thus, $2 a u=2$, and the proof is complete.

The next result was obtained independently by $R$. Hartwig and J. Luh in [6].

Proposition 3.7. A ring $R$ with identity is a division ring or a Boolean ring if and only if each nonzero element of $R$ has a unique invertible generalized inverse.

Proof. In a division ring each nonzero element is invertible, and in a Boolean ring the identity is the only invertible element; hence, the necessity is clear. Conversely, suppose the condition holds and $R$ is not a division ring. By Lemmas 3.6 and 3.3, $R$ has a nontrivial central idempotent, so $R=S \oplus T$ where $S$ and $T$ are nonzero rings with identities $e$ and $f$, respectively. If $u$ is any unit in $T$, then $(e, u)$ is an invertible generalized inverse of $(e, 0)$ in $R$. Hence, $T$ has a unique invertible element. Similarly, $S$ has a unique invertible element. Thus, 1 is the unique invertible element of $R$. Since each element of $R$ is unit regular, each element is consequently idempotent. Therefore $R$ is Boolean.

Recall that a ring $R$ has no integral torsion if whenever $a$ is in $R$ and $n$ is an integer then $n a=0$ implies $n=0$ or $a=0$. 
Proposition 3.8. Suppose $R$ is a ring with no integral torsion and $a$ is a regular element of $R$. Then each of the following statements holds:

(i) $R$ has an identity and $a$ is a unit or a has infinitely many generalized inverses.

(ii) a has a unique reflexive inverses or infinitely many reflexive inverses.

(iii) If, in addition, $R$ is regular with identity and $a$ is unit regular, then $a$ is a unit or a has infinitely many invertible generalized inverses.

Proof. (i) Let $M$ denote the set of generalized inverses of $a$ and suppose $z$ is an element of $R$ for which $a z=0$ or $z a=0$. Let $N=\{x+z: x \in M\}$. Then $M$ contains $N$ and the map sending $x$ to $x+z$ is a bijection of $M$ onto $N$. Hence, if $M$ is finite, then $M=N$. If we write $M=\left\{x_{1}, \cdots, x_{m}\right\}$, then there is a permutation $\pi$ of the indices $1, \cdots, m$ such that $x_{i}=x_{i \pi}+z$ for $1 \leqq i \leqq m$. If $n$ is the length of the orbit of 1 under $\pi$, then it follows that $x_{1}=$ $x_{1}+n z$. Since $n \geqq 1$ and $R$ has no integral torsion, we deduce $z=$ 0 . Thus, $a$ is neither a right nor a left divisor of zero, so is a unit by Lemma 3.1.

(ii) If $a$ does not have a unique reflexive inverse, then, by Lemma 3.3, there is an $x$ in $R$ such that $a=a x a$ and $a x \neq x a$. By passing to the element $x a x$ if necessary, we can assume $x$ is a reflexive inverse of $a$. Now at least one of the elements $a x-x a^{2} x$ and $x a-x a^{2} x$ is not zero, for otherwise $a x=x a$. Say $a x-x a^{2} x \neq$ 0 . It is now routine to check that $\left\{x+n\left(a x-x a^{2} x\right): n=1,2, \cdots\right\}$ is an infinite set of reflexive inverses of $a$.

(iii) First note that for a nonzero integer $n, n=n \cdot 1$ is not a zero divisor in $R$, so is a unit by Lemma 3.1. Now let $U$ denote the set of invertible generalized inverses of $a$ and assume $U$ is finite. By Lemma 3.5, $a$ has only finitely many reflexive inverses and hence, by (ii) above, $a$ has a unique reflexive inverse $x$. If $u$ is in $U$, then $u a u$ is a reflexive inverse of $a$ and so $x=u a u$. From this relation follows $x u^{-1}=u a=x a$ and, consequently $u+x$ is a unit with inverse $u^{-1}-2^{-1} a$. Thus, $u+x$ is a unit for all $u$ in $U$. It follows that the map sending $u$ to $2^{-1}(u+x)$ is a one-one map of $U$ into itself. Since $U$ is finite, the map is onto. If we write $U=\left\{u_{1}, \cdots, u_{m}\right\}$, then there is a permutation $\pi$ of the indices such that $u_{i}=2^{-1}\left(u_{i \pi}+x\right)$ for $1 \leqq i \leqq m$. If $n$ is the length of the orbit of 1 under $\pi$, then $u_{1}=2^{-1}\left(u_{1 \pi}+x\right)=\cdots=2^{-n}\left[u_{1}+(2 n-1) x\right]$. Hence, $x=\left(2^{n}-1\right)(2 n-1)^{-1} u_{1}$ is a unit of $R$. Since $x=x a x$, it follows that $a$ is a unit of $R$ as well. This completes the proof.

In the sequel we follow the usual conventions concerning the 
formal use of 1 in a ring $R$ without identity. For example, $a R(1-$ $b$ ) denotes the set of all elements of the form $a r-a r b$. Further, if $R$ lacks an identity, then the phrase " $\alpha$ is a nonunit of $R$ " means " $a$ is any element of $R$."

The next two results are preparatory to the theorem on prime regular rings, mentioned in the introduction.

Lemma 3.9. Suppose $R$ is a prime ring with elements a and $x$ such that $a=a x a$. If there is a nonzero $b$ in $R$ such that $b R(1-a x)=(0)$, then $R$ has an identity and a has a right inverse in $R$.

Proof. With $e=a x$, the hypothesis implies $b R(r-r e)=(0)$ for every $r$ in $R$. Since $R$ is prime and $b \neq 0$, it follows that $e$ is a right identity of $R$. But in a prime ring a right identity is also a left identity, so $e=a x$ is the identity of $R$, and the lemma is proven.

LEMMA 3.10. Suppose $R$ is a ring with identity and $a, p, q \in R$ with $p, q$ units in $R$. Then $a$ and paq have the same number of generalized inverses, of reflexive inverses, and of invertible generalized inverses.

Proof. If $X$ and $Y$ denote the sets of inverses of one type for $a$ and $p a q$, respectively, then the map sending $x$ to $q^{-1} x p^{-1}$ is a bijection of $X$ onto $Y$.

THEOREM 3.11. Suppose $R$ is a prime regular ring which is not a division ring. Then the following statements are equivalent:

(i) $R$ has infinitely many elements.

(ii) Every nonunit of $R$ has infinitely many generalized inverses.

(iii) Every nonzero nonunit of $R$ has infinitely many reflexive inverses.

If, in addition, $R$ has an identity, then each of these statements is equivalent to

(iv) Every unit regular nonunit of $R$ has infinitely many invertible generalized inverses.

Proof. Since $R$ is not a division ring, each of (ii) and (iv) implies (i), and (iii) implies (ii). To see that (iii) implies (iv), note that any nonzero, unit regular nonunit must have infinitely many invertible generalized inverses by Lemma 3.5. Since $R$ is not a division ring, it follows that $R$ has an infinite set of units, so (iv) 
holds for the zero element as well. To complete the proof of the theorem, we need only show (i) implies (iii).

If (iii) does not hold, then $R$ contains a nonzero nonunit $a$ which has only a finite number of reflexive inverses. Note that $a$ does not have a right inverse, for otherwise it would have infinitely many right inverses by Kaplansky's theorem, and hence, infinitely many reflexive inverses. Let $x$ be any reflexive inverse of $a$.

Suppose $\left\{e_{n}\right\}$ is an infinite sequence of orthogonal idempotents in $R$, and assume $e_{n} R \cap x a R \neq(0)$ for infinitely many $n$. Since $x a$ is idempotent, this assumption means that there are $y_{n}$ in $R$ such that $e_{n} y_{n}=(x a)\left(e_{n} y_{n}\right) \neq(0)$ for infinitely many $n$. By Lemma 3.9, there are $z_{n}$ in $R$ such that $e_{n} y_{n} z_{n}(1-a x) \neq 0$ for infinitely many $n$. Since the $e_{n}$ 's are orthogonal, the set $\left\{e_{n} y_{n} z_{n}(1-a x): n=1,2, \cdots\right\}$ is infinite. Therefore, the set $\left\{x+e_{n} y_{n} z_{n}(1-a x): n=1,2, \cdots\right\}$ is infinite as well. But every element of the last set is easily seen to be a reflexive inverse of $a$. This contradiction shows that $e_{n} R \cap x a R=(0)$ for all but finitely many $n$. Similarly, if $e_{n} R \cap(1-$ $x a) R \neq(0)$ for infinitely many $n$, then there are $w_{n}$ in $R$ such that $e_{n} w_{n} a x \neq 0$ and $a\left(e_{n} w_{n}\right)=0$ for infinitely many $n$. Hence, $\{x+$ $\left.e_{n} w_{n} a x: n=1,2, \cdots\right\}$ is an infinite set of reflexive inverses of $a$. From this contradiction and the previous result obtained, we deduce

$$
e_{n} R=\left(e_{n} R \cap x a R\right) \oplus\left(e_{n} R \cap(1-x a) R\right)=(0)
$$

for all but finitely many $n$. Thus, $e_{n}=0$ for all but finitely many $n$, and therefore $R$ has no infinite sequence of nonzero orthogonal idempotents. By Kaplansky's theorem [11, Theorem 2.1], it follows that $R$ is Artinian.

By the Wedderburn-Artin theorem, $R$ is the $m \times m$ matrix ring over some division ring $D$. If the matrix $a$ has rank $k$, then the fact that $a$ is a nonzero nonunit guarantees that $1 \leqq k<m$. There are units $p$ and $q$ in $R$ such that $p a q=\operatorname{diag}(1, \cdots, 1,0, \cdots, 0)$, where 1 appears $k$ times. By Lemma 3.10, $a$ has the same number of reflexive inverses as paq. But any $m \times m$ matrix of the form

$$
\left[\begin{array}{cc}
I_{k} & X \\
Y & Y X
\end{array}\right]
$$

where $I_{k}$ is the $k$ by $k$ identity matrix, $X$ is any $k$ by $(m-k)$ matrix, and $Y$ is any $(m-k)$ by $k$ matrix, is a reflexive inverse of paq. Therefore $D$ is finite and hence $R$ is finite as well.

It is well-known that a finite regular ring is a direct sum of finitely many full matrix rings over finite fields. This results from the semisimplicity of a regular ring, the Wedderburn-Artin theorem, and the Wedderburn theorem on finite division rings. (See 
J. Dyer-Bennet [3].) Thus, we can restate Theorem 3.11 in the following form: If a prime regular ring contains an element for which the number of generalized inverses (or reflexive inverses, or invertible generalized inverses) is finite and greater than one, then $R$ is a full matrix ring over a finite field.

Our next aim is to characterize those regular rings for which each element possesses only finitely many generalized inverses of each type. The proof to the first result in this direction is elementary.

THEOREM 3.13. A nonzero regular ring $R$ is a division ring or a finite ring if and only if each nonzero element of $R$ has only finitely many generalized inverses.

Proof. The necessity is clear. Conversely, suppose the condition holds and $R$ is not a division ring. Let $e$ be a nontrivial idempotent in $R$ and express $R$ in the Peirce decomposition

$$
R=e R e \oplus(1-e) R(1-e) \oplus e R(1-e) \oplus(1-e) R e .
$$

Now if $a$ is any nonzero element of $R$ and if $a=a x a$, then the set $\{x+z: z a=0$ or $a z=0\}$ is contained in the set of generalized inverses of $a$ and is therefore finite. Consequently, each nonzero element of $R$ has only finitely many left or right annihilators. Since each summand in the above Peirce decomposition left or right annihilates each other summand, it follows that each summand, and hence $R$, is finite.

We now consider the case in which each element of a regular ring has only finitely many reflexive inverses. Such a ring need not have bounded index of nilpotency. For example, let $F_{n}$ denote the $n \times n$ matrix ring over a finite field $F$ and let $P$ denote the direct product of the family $\left\{F_{n}\right\}$. Let $R$ consist of all $f$ in $P$ for which there is a positive integer $N$ and a $c$ in $F$ such that $f_{n}=c \cdot l_{n}$ for all $n \geqq N$, where $1_{n}$ is the identity in $F_{n}$. Each element of $R$ has only finitely many reflexive inverses because each coordinate in the initial finite segment has only finitely many and the tail segment has a unique one. Our next theorem says that this example is essentially typical. We first record a version of McCoy's lemma $[10$, p. 111].

LeMma 3.13. Let $\psi: R \rightarrow S$ be an epimorphism of regular rings and let $a$ be an element of $R$. Let $X$ be the set of reflexive inverses of $a$ in $R$ and $Y$ the set of those of $a \psi$ in $S$. Then $X \psi=Y$.

Proof. Suppose $z \psi^{\prime} \in Y$. Then $a-a z a$ is in the kernel of $\psi$ 
(which is a regular ring), so there is a $k \in R$ such that $a=a(z+$ $k) a a$ and $k \psi=0$. Let $x=(z+k) a(z+k)$. Then $x$ is a reflexive inverse of $a$ and $x \psi=z \psi$. Thus, $Y \leqq X \psi$ and, as the reverse inclusion is clear, the lemma is proven.

THEOREM 3.14. For a regular ring $R$ the following statements are equivalent:

(i) Each element of $R$ has only finitely many reflexive inverses.

(ii) Every prime factor ring of $R$ is a division ring or a finite ring, and for each $a$ in $R$ the set of prime ideals $P$ for which $a+P$ is a nonzero nonunit of $R / P$ is finite.

Proof. Suppose (ii) holds and $a \in R$. If $a+P$ is zero or a unit in $R / P$ for all primes $P$, then $a$ has a unique reflexive inverse in $R$, by Lemma 3.3 and the fact that $R$ is semiprime. Otherwise, let $P_{1}, \cdots, P_{n}(n \geqq 1)$ be a list of all distinct prime ideals $P$ for which $a+P$ is a nonzero nonunit of $R / P$, and let $I$ be the intersection of the $P_{i}$ 's. By the Chinese remainder theorem [10, p. 109], $R / I$ is the direct sum of the rings $R / P_{i}$. Since $a+P_{i}$ has only finitely many reflexive inverses in $R / P_{i}$, the element $a+I$ has only finitely many reflexive inverses in $R / I$. By Lemma 3.13, there are reflexive inverses $x_{1}, \cdots, x_{m}$ of $a$ in $R$ such that $x_{1}+I, \cdots, x_{m}+I$ is a complete list of reflexive inverses of $a+I$ in $R / I$. Since the image of $a$ in each other prime factor ring has a unique reflexive inverse and since $R$ is semiprime, it is clear that $x_{1}, \cdots, x_{m}$ is a complete list of reflexive inverses of $a$ in $R$.

Conversely, suppose condition (i) holds. By Lemma 3.13 and Theorem 3.11, every prime factor ring of $R$ is a division ring or a finite ring. To prove the second part of (ii), let $a$ be in $R$ and suppose $P_{1}, \cdots, P_{n}$ are distinct primes in $R$ for which $a+P_{i}$ is a nonzero nonunit of $R / P_{i}$. Let $m_{i}$ denote the number of reflexive inverses of $a+P_{i}$ in $R / P_{i}$. By Lemma 3.3 and the fact that a prime ring has no nontrivial central idempotents, each $m_{i}>1$. The number of reflexive inverses of the image of $a$ in $R / P_{1} \oplus \cdots \oplus R / P_{n}$ is $m_{1} m_{2} \cdots m_{n}$. By the Chinese remainder theorem and Lemma 3.13, $a$ has at least $m_{1} m_{2} \cdots m_{n}$ reflexive inverses in $R$. The second assertion in (ii) is now clear.

COROLLARY 3.15. For a regular ring $R$ the following statements are equivalent:

(i) There is a positive integer $N$ such that every element of $R$ has at most $N$ reflexive inverses.

(ii) $R$ is the direct sum of a strongly regular ring and a 
finite ring.

Proof. By Proposition 3.4, statement (ii) clearly implies statement (i). Conversely, if (i) holds then every prime factor ring of $R$ is a division ring or a finite ring by Theorem 3.14. Arguing as in the proof to the sufficiency of Theorem 3.14, it follows that $R / P$ is a division ring for all but finitely many primes $P$. Let $I$ be the intersection of those $P$ for which $R / P$ is a division ring and $J$ the intersection of the finitely many remaining $P$. Then $R / I$ is strongly regular, $R / J$ is finite, and $R=R / I \oplus R / J$ by another application of the Chinese remainder theorem.

Next we characterize those unit regular rings for which each nonzero element has only finitely many invertible generalized inverses. For this purpose we note

LEMMA 3.16. If $\psi: R \rightarrow S$ is an epimorphism of unit regular rings, then $\psi$ takes the group of units of $R$ onto the group of units of $S$.

Proof. If $a \psi$ is a unit of $S$ and $a=a u a$ where $u$ is a unit of $R$, then $a \psi=u^{-1} \psi$.

THEOREM 3.17. For a regular ring $R$ with identity, the following statements are equivalent:

(i) Each nonzero element of $R$ has at least one and at most finitely many invertible generalized inverse (s).

(ii) $R$ is a division ring or the group of units of $R$ is finite.

(iii) $R$ is a division ring, a Boolean ring, a finite ring, or the direct sum of a Boolean ring and a finite ring.

Proof. That (iii) implies (ii) is clear.

(ii) implies (i). Assume (ii) is true. Then every unit regular element of $R$ has only finitely many invertible generalized inverses. So, to establish (i), we need only show that $R$ is unit regular. Suppose $P$ is a prime ideal of $R$ and $R / P$ is not a division ring. Then there is an idempotent $e$ in $R$ such that $e+P$ is a nonzero nonunit of $R / P$ and, hence, the number of reflexive inverses of $e+P$ in $R / P$ is larger than one. Since $e+P$ is unit regular in $R / P$, the number of reflexive inverses of $e+P$ in $R / P$ is finite, by Lemmas 3.16 and 3.5. By Theorem 3.11, the prime ring $R / P$ is finite. Thus, every prime factor ring of $R$ is Artinian, so $R$ is unit regular by Fisher-Snyder [5, Theorem 1.1].

(i) implies (iii). Suppose (i) is true. By Lemma 3.5, each element of $R$ has only finitely many reflexive inverses. By Theorem 
3.14, every prime factor ring of $R$ is a division ring or a finite ring. If the center of $R$ is a field, then $R$ is simple by [9, Theorem 3 , p. 239], in which case $R$ is a division ring or a finite ring, and so (iii) holds. If the center of $R$ is not a field, then $R$ has a nontrivial central idempotent by Lemma 3.3. Hence, we can write $R=S \oplus T$, where $S$ and $T$ are nonzero rings with identities. If $e$ is the identity for $S$ and $u$ is any unit of $T$, then $(e, 0)=(e, 0)(e$, $u)(e, 0)$ and $(e, u)$ is a unit of $R$. Since $(e, 0)$ has only finitely many invertible generalized inverses in $R$, it follows that $T$ has only finitely many units. By the same token, $S$ has only finitely many units. Hence, the group of units of $R$ is finite. By Lemma 3.16, the group of units in each prime factor ring of $R$ is finite. Since each prime factor ring of $R$ is a division ring or a finite ring, it follows that each prime factor ring of $R$ is finite. If $\left\{P_{i}\right\}$ is an infinite sequence of prime ideals of $R$ for which $R / P_{i}$ is not the two-element field, then the number of units in $R / P_{1} \oplus \cdots \oplus R / P_{n}$ increases as $n$ increases, since each $R / P_{i}$ is a full matrix ring over a field. By the Chinese remainder theorem and Lemma 3.16, this implies $R$ has an infinite set of units. This contradiction shows that $R / P$ is the two-element field for all but finitely many primes $P$.

There are now three cases to consider. If every prime factor ring of $R$ is the two-element field, then $R$ is Boolean. If no prime factor ring of $R$ is the two-element field, then $R$ is a finite ring. In the final case, $R$ is the direct sum of a Boolean ring and a finite ring by another application of the Chinese remainder theorem.

\section{REFERENCES}

1. R. Arens and I. Kaplansky, Topological representations of algebras, Trans. Amer. Math. Soc., 63 (1948), 457-481.

2. C. W. Bitzer, Inverses in rings with unity, Amer. Math. Monthly, 70 (1963), 315.

3. J. Dyer-Bennet, On finite regular rings, Bull. Amer. Math. Soc., 47 (1941), 784-787.

4. G. Ehrlich, Unit regular rings, Portugal Math., 27 (1968), 209-212.

5. J. Fisher and R. Snider, Rings generated by their units, J. Algebra, 42 (1976), 363-368.

6. R. Hartwig and J. Luh, A note on the group structure of unit regular ring elements, Pacific J. Math., 71 (1977), 449-461.

7. M. Henriksen, On a class of regular rings that are elementary divisor rings, Arch. Math., 24 (1973), 133-141.

8. N. Jacobson, Some remarks on one-sided inverses, Proc. Amer. Math. Soc., 1 (1950), 352-355.

9. - Structure of Rings, Amer. Math. Coll. Publ. XXXVII, Providence, 1964.

10. I. Kaplansky, Fields and Rings, Univ. of Chicago Press, Chicago, 1972.

11. - Topological representations of algebras II, Trans. Amer. Math. Soc., 68 (1950), 62-75.

12. N. McCoy, Theory of Rings, Chelsea Publ. Co., Bronx, 1973.

13. J. von Neumann, On regular rings, Proc. Nat. Acad. Sci., 22 (1936), 707-713. 
Received May 1, 1978 and in revised form October 13, 1979.

St Olaf College

NORTHFIELD, MN 55057 
.././. ./FrontMatter/paper .pdf 


\section{Pacific Journal of Mathematics \\ Vol. 87, No. $2 \quad$ February, 1980}

Theagenis Abatzoglou, Unique best approximation from a $C^{2}$-manifold in Hilbert space ................................. 233

Gerald Arthur Anderson, $\Lambda$-homology cobordism bundles............. 245

Eric Bedford, Holomorphic mapping of products of annuli in $\mathbf{C}^{n} \ldots \ldots \ldots 271$

Gunnar Carlsson, On the stable splitting of $b o \wedge b o$ and torsion operations in connective $K$-theory .......................... 283

Lester Eli Dubins and David Samuel McIntyre Margolies, Naturally integrable functions ................................. 299

Leo Egghe, The Radon-Nikodým property, $\sigma$-dentability and martingales in locally convex spaces ............................. 313

Irving Leonard Glicksberg, Maps preserving translates of a function ..... 323

Hugh M. Hilden and Robert D. Little, Cobordism of branched covering

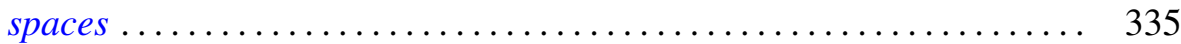

Russell Allan Johnson, Almost-periodic functions with unbounded integral .......................................... 347

Bruce Stephen Lund, The endomorphisms of a Dirichlet algebra ........ 363

John Henry McCleary, Mod $p$ decompositions of H-spaces; another approach........................................ 373

Arlan Bruce Ramsay, Subobjects of virtual groups ................ 389

Thomas R. Savage, Generalized inverses in regular rings ............ 455 Jaak Vilms, On curvature operators of bounded rank ......... 